\title{
Automation, operation, and data analysis in the cryogenic, high accuracy, refraction measuring system (CHARMS)
}

\author{
Bradley J. Frey", Douglas B. Leviton \\ NASA Goddard Space Flight Center, Greenbelt, MD 20771
}

\begin{abstract}
The Cryogenic High Accuracy Refraction Measuring System (CHARMS) at NASA's Goddard Space Flight Center has been enhanced in a number of ways in the last year to allow the system to accurately collect refracted beam deviation readings automatically over a range of temperatures from $15 \mathrm{~K}$ to well beyond room temperature with high sampling density in both wavelength and temperature. The engineering details which make this possible are presented. The methods by which the most accurate angular measurements are made and the corresponding data reduction methods used to reduce thousands of observed angles to a handful of refractive index values are also discussed.
\end{abstract}

Keywords: Cryogenic, refractive index, infrared, refractometer, NIRCam, CHARMS, high accuracy, automation

\section{INTRODUCTION}

The Cryogenic High Accuracy Refraction Measuring System (CHARMS) is a minimum deviation refractometer at the NASA / Goddard Space Flight Center (GSFC) capable of measuring the absolute refractive index (i.e. in vacuum) of optical materials over a wide range of environmental conditions. CHARMS currently measures absolute refractive index from $0.4 \mu \mathrm{m}$ to $5.6 \mu \mathrm{m}$ in wavelength and at temperatures as low as $15 \mathrm{~K}$. This system has been under development for more than four years and our incremental development has been previously documented ${ }^{1,2,3}$. The intention of this text is to summarize the improvements made to the system hardware and to describe the automated data acquisition and data reduction processes. A companion paper ${ }^{4}$ describes in detail the cryogenic refractive index data we have gathered on $\mathrm{BaF}_{2}, \mathrm{LiF}$, and ZnSe in support of the James Webb Space Telescope (JWST) / Near-Infrared Camera (NIRCam).

\section{HARDWARE UPGRADES AND DATA ACQUISITION PROCEDURES}

The hardware implementation in CHARMS has been evolving nearly continuously since its inception, with the ultimate goal being the development of the most robust and accurate refractometer possible. Other considerations include ease of computer control and system automation as well as improving the overall efficiency of the data acquisition. Under these careful considerations, we have been able to improve the system performance several fold: from the laborious, manual data acquisition at a rate of almost $6 \mathrm{~min} /$ data point to the nearly continuous and autonomous (with the exception of cryogen management) rate of $2 \mathrm{~min} /$ data point with the capability to perform measurements unattended 24 hours a day, 7 days a week. When a typical dataset for a single sample material consists of more than 3000-4000 individual data points, these are important considerations.

Three of the most important factors that determine the overall accuracy of the refractive index data generated by CHARMS are: accurate measurement of the angle through which refracted rays are deviated by the sample prism, knowledge of sample temperature, and knowledge of incident wavelength. We have upgraded the hardware required to accurately measure all three of these factors, and we present those details below.

\subsection{Drive mechanism for rotating fold mirror}

Inherent in the accuracy of CHARMS is the ability to accurately manipulate and measure the position of two high precision rotation stages. These custom stages have extremely low radial runout and are outfitted with absolute optical encoders capable of reporting rotational positions at the sub-arcsecond level ${ }^{5}$. In order to utilize the capabilities of these stages in positioning our sample prism and rotating fold mirror, we need an equally high caliber drive mechanism.

\footnotetext{
*Brad.Frey@nasa.gov, phone 1-301-286-7787, FAX 1-301-286-0204
} 
Initially, we had implemented a drive system consisting of a vacuum compatible stepper motor with a belt and pulley arrangement simply wrapped around the worktable of our rotation stages. ${ }^{3}$ We implemented this system on both the sample prism rotation stage and the rotating fold mirror rotation stage, with the addition of a "step-down" gear-reducing pulley in the rotating fold system to improve resolution. While this system was simple, inexpensive, and worked well, it presented several challenges. As could be expected with such a belt drive system, there was significant windup and backlash as tension was applied to the belts by the stepper motors. In addition, the stages would continue to drift as the belts relaxed after the intended motion was complete. To remedy these inadequacies, we replaced the belt drive system on the rotating fold mirror stage with a tangent arm arrangement driven through a single point of contact by a linear translation stage comprised of a (different) stepper motor and lead screw (see Figure 1). This combination has allowed us to maintain the resolution requirement on our rotations and still get adequately accurate refractive index measurements, but without the complications that plagued the belt drive system. Since the rotations of the sample prism are smaller and less frequent, and the requirement on their accuracy less stringent than that on the rotating fold mirror, we have not needed to replace the stepper motor/belt drive on the sample prism rotation stage.

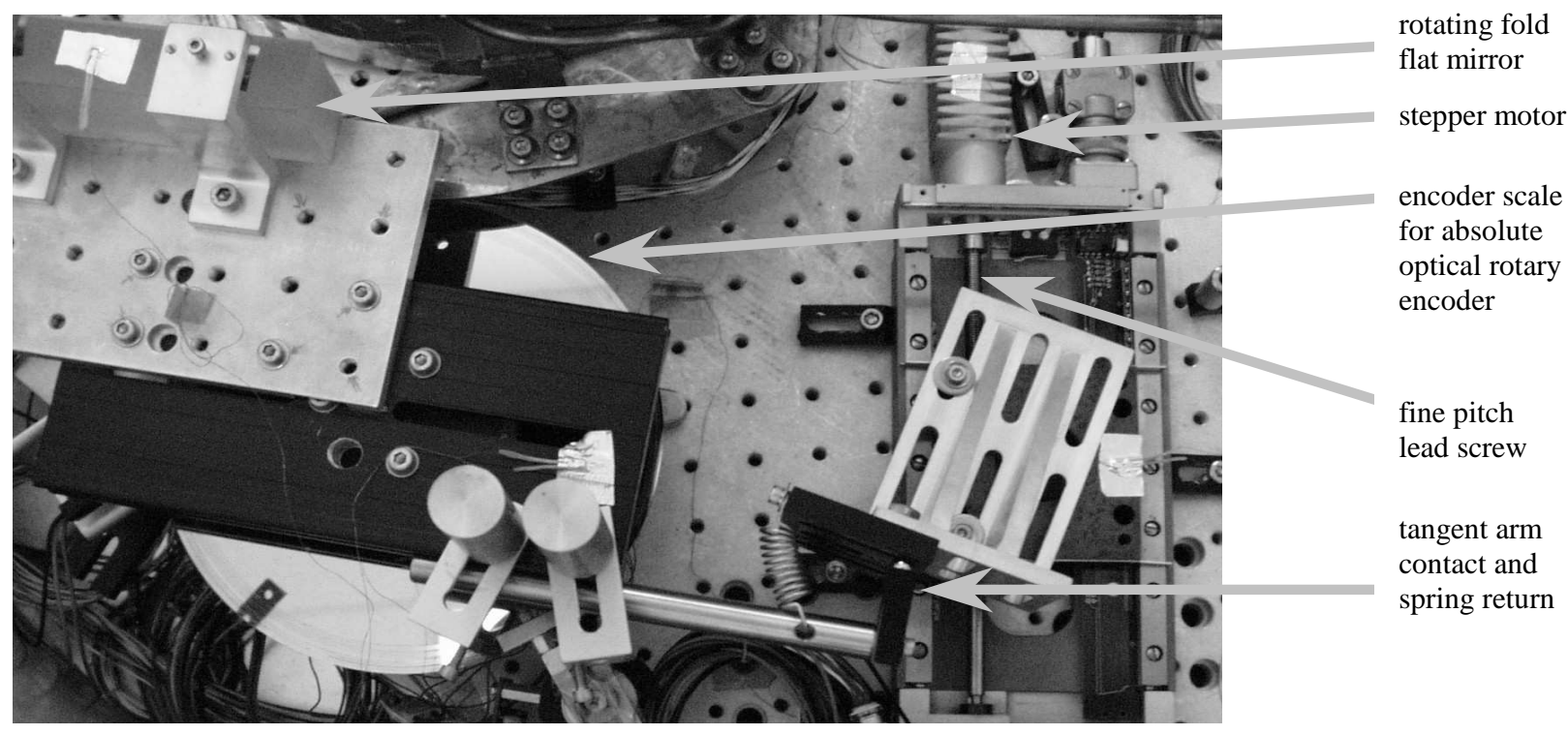

Figure 1: Tangent arm drive mechanism used to accurately rotate fold mirror. The tangent arm utilizes a single point of contact between a stainless steel ball bearing and flat plate. A spring return maintains ball and plate contact at all times during the motion.

\subsection{Determination of deviation angle}

The angles through which the refracted rays are deviated by the sample prism are measured by the high precision rotation stages and optical encoders, used in combination with one of our detector arrays at a time. In order to make measurements over the spectral range 0.4 to $5.6 \mu \mathrm{m}$, we use a combination of as many as three detectors: a CCD $(0.4$ to $1.15 \mu \mathrm{m})$, an InSb array $(1.1$ to $5.6 \mu \mathrm{m})$, and an InGaAs array $(0.9$ to $1.7 \mathrm{um}$ - to date used primarily for diagnostic purposes). The method by which these detectors have been used to determine deviation angles has evolved based on measurement accuracy, data acquisition rate, and ease of automation requirements.

For a given detector, the deviation angle of a sample prism in CHARMS is defined as the difference in the angle between the rays deviated by the sample prism and those left undeviated. The absolute angle assigned to the deviated (or undeviated) beam is the angle reported by the optical encoders on the rotating fold mirror stage when the centroid of the slit image of the deviated (or undeviated) beam lands on a pre-defined reference column of the detector array. In practice, the determination of the centroid position of the slit image varies for different detectors as described below. Common to the deviation angle determination for all methods, however, is the requirement to properly set and subsequently maintain the condition of minimum deviation.

Critically establishing the condition of minimum deviation (CMD) is important in that once it has been established, we can easily calculate the absolute refractive index given only the apex angle of the prism and the deviation angle for a 
given wavelength and temperature according to:

$$
\mathrm{n}_{\mathrm{abs}}(\lambda, \mathrm{T})=\sin ((\alpha(\mathrm{T})+\delta(\lambda, \mathrm{T})) / 2) / \sin (\alpha(\mathrm{T}) / 2)
$$

where $\alpha$ is the apex angle of the prism and $\delta$ is the angle through which light of wavelength $\lambda$ is deviated by a sample prism at temperature $\mathrm{T}$. If that condition is not properly set (or maintained over the course of a dataset), we will necessarily introduce a systematic error that will result in measured indices which are always higher that those expected. Fortunately, the required precision for properly setting and maintaining CMD is not exceedingly difficult to achieve and in practice the contribution of lack of CMD maintenance to our overall error budget is negligible.

The condition of minimum deviation is established as follows: with the slit image on the array, the sample prism is rotated such that the slit image walks across the array detector toward angles of smaller deviation. As we approach and subsequently pass through the CMD by monotonically rotating the sample prism, the slit image will slow to a stop, then turn around and walk in the opposite direction across the detector (to angles of larger deviation, hence the term "condition of minimum deviation"). During this process on either side of CMD, we record several (slit image centroid, sample prism encoder position) ordered pairs which are then averaged together to determine what the sample prism encoder would have read when the prism was at the condition of minimum deviation. This process (albeit incorrectly) assumes the function of deviation angle vs. incident angle is symmetric about the CMD and therefore will introduce a systematic error into our measurements. However, this translates into an error in overall refractive index several orders of magnitude smaller than our system's stated accuracy and is therefore negligible.

CMD has now been established (once per dataset), but it is also a function of temperature and wavelength. We must therefore be careful to maintain CMD at every one of the thousands of data points that make up a dataset. This is done for each data point by first moving the slit image to the reference column of the detector. The software then compares the current value of the optical encoder on the rotating fold mirror stage with that value defined when CMD was initially established. This offset in rotating fold position is analytically the same offset through which the sample prism will need to be rotated to re-establish CMD $^{6}$. This process is iterated, but in general deviation from CMD is such a slowly changing function that a single iteration is sufficient.

Once CMD has been adequately established, the automated measurement of the deviation angle may commence. For data acquired by the CCD (and the InGaAs array), the reference column is the center column of pixels on the detector. To determine the angular orientation of the rotating fold flat that is required to position the centroid of the slit image on this reference column, we could simply iterate the position of the mirror until the centroid is within some tolerance (and within some measurement uncertainty) of the reference column. We have found that iterative process to be too time consuming and the limit of our ability to accurately determine the centroid value dominated the noise in the resulting index data. Alternatively, we found that taking multiple centroid values (along with corresponding fold flat encoder readings) at various locations across the detector array straddling the reference column gave us better results. Because the specific centroid locations on the detector are arbitrary, using this method (as long as we have sufficient knowledge of what they are) there is no need for iteration. By measuring a few - typically 4 - (centroid, encoder) ordered pairs ( 2 on either side of the reference column) and performing a least-squares fit to a line passing through them, we can very precisely and accurately solve for what the fold flat angle would have been at the reference column.

For the InSb detector we had historically used a similar approach, but we have devised another method that has proven itself superior. Since the InSb array is sensitive to IR radiation, it is of course a good thermometer of the optical components by which it sees signal light. As we make refractive index measurements as a function of temperature, the background level in the images recorded by the InSb array will therefore change as well. This changing background due to sub-Kelvin changes in temperature lead to an unfavorable (and dynamic) signal over background level on timescales roughly equal to those of a single data point. As a consequence, we were forced to limit our exposure times and increase the frequency on which we performed (time-consuming) background corrections. This was only exacerbated by the fact that we now needed to co-add multiple image frames to build up sufficient signal over background levels.

Instead, we decided to fabricate a $150 \mu \mathrm{m}$ x $3 \mathrm{~mm}$ slit in a $25 \mathrm{~mm}$ diameter aluminum disk. We mounted this detector slit in the cold stop (cooled along with the array to $77 \mathrm{~K}$ ) of the InSb array and remounted the camera such that the detector slit was now in the focal plane of our refractometer camera mirror. Thus, this disc dramatically reduces the 
view factor of the InSb array to only a small area and consequently provides a lower and more stable image background level. This in turn allows us to use longer exposure times and fewer co-adds, ultimately reducing measurement time.

With this detector slit installed, we can obviously no longer use this detector as an image sensor as our ability to discern spatial information has been lost. In contrast, we now use the array essentially as a large, multi-element radiometer. As we step the monochromator slit image across this detector slit, we measure the integrated flux passing through the detector slit at each step. We simultaneously record fold flat encoder rotation angle readings so that we can very accurately map out this intensity distribution and therefore derive the encoder reading corresponding to when the slit image centroid was centered on the detector slit. We repeat this procedure for both the deviated and undeviated beams. The detector slit center position has effectively replaced our array detector "reference column."

Further, this net decrease in measurement time has allowed us to extend the useful wavelength response range of the InSb down to $1.1 \mu \mathrm{m}$ to overlap with measurements obtained using the CCD. While the InSb array has always been sensitive at this - and shorter - wavelengths, measurements in the NIR have always been plagued by a detector "burning" effect ${ }^{3}$ : a localized, temporary deadening of pixels after being exposed to radiation at wavelengths of $1.3 \mu \mathrm{m}$ and shorter. By limiting the amount of time that the detector is exposed to such radiation, we have been able to mitigate the "burning" effect to a tolerable level so that we can still perform measurements at these wavelengths. Extending this useful measurement range has proven to be an excellent diagnostic and system characterization tool.

To verify the accuracy of this new data acquisition method, we performed two different tests. First, we compared results of our measurements of fused silica with those measured by I.H. Malitson ${ }^{7}$, widely accepted to be a standard candle in refractive index measurements. Second, we compared our measurements of several different materials (fused silica, $\mathrm{LiF}$, $\mathrm{BaF}_{2}$, and $\mathrm{ZnSe}$ ) taken with the $\mathrm{CCD}, \mathrm{InGaAs}$, and InSb detectors at wavelengths for which the three detectors overlap. In both cases, the data were shown to be in agreement at or below our derived system accuracy for each of the respective materials. After completing this characterization, we believe we have implemented a superior data acquisition method not only in terms of data accuracy but also in system measurement efficiency.

\subsection{Temperature sensor mounting}

Knowledge of sample temperature is the second critical factor required to make accurate refractive index measurements. Depending on the magnitude of the thermo-optic coefficient (dn/dT) for a given material, uncertainty in knowledge of temperature can be the leading source of uncertainty in our overall error budget for CHARMS. Determination of sample temperature is made by three independent calibrated silicon diodes that are accurate to better than $0.03 \mathrm{~K}$ over the operating range of CHARMS. Two of these sensors are mounted at different locations on the top of the sample prism (one near the apex, one near the non-refracting face), and the third near the bottom of the non-refracting face (see Figure 2). While we do see thermal gradients of as much as several degrees while we are actively cooling the sample (during which time we do not perform measurements), the three sensors generally agree at the sub-Kelvin level once the sample has reached equilibrium and are therefore averaged to determine the "measured" sample temperature.

Originally, we had temporarily bonded the diodes to the sample prism using commercially available "varnish" that could easily be removed with a small amount of acetone. This allowed us to non-destructively attach the diodes directly to the prism faces without the need for additional hardware that could inadvertently introduce a mechanical or thermal interference with existing hardware in our (already tightly constrained) sample chamber. Seemingly having found an ideal thermometry means, we embarked on our first sets of measurements. We soon discovered though, that after repeated thermal cycling, the bond between the sensor and sample prism began to degrade as evidenced by a growing disagreement at the coldest temperatures between the readings reported by the three sensors as they were thermally cycled. The phenomenon was likely due to a CTE mismatch between the sample prism, varnish, and/or sensor as they were cooled, especially since it was severe for some sample materials and almost negligible for others, and the effect disappeared as the sample prism approached room temperature. Rebonding the sensors temporarily resolved the issue, but we would again see a divergence of sensor readings after repeated thermal cycles (see Figure 10, Section 4).

In order to alleviate the uncertainties associated with inconsistent sensor attachment, we adapted a commercially available, spring-loaded sensor mount to be compatible with our sample chamber geometry for two of the three sensors (see Figure 2). For the third sensor on the non-refracting face of the sample prism, we employed the same spring-loaded mount concept, but slightly modified it to minimize the interference with the undeviated light beam that passes in close 


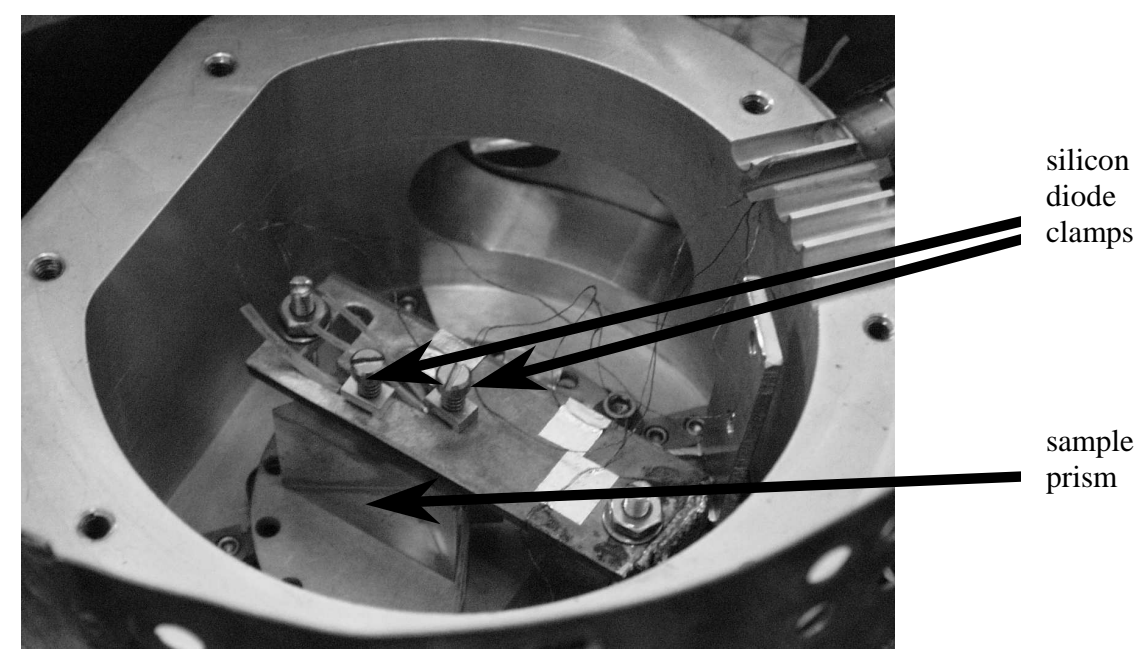

Figure 2: Sample prism in LHe cooled windowless sample chamber. Two calibrated silicon diodes are mounted on the top of the sample prism using spring-loaded clamps, a third is similarly mounted on the non-refracting face (not shown).

proximity. This type of mechanical arrangement avoids CTE mismatch effects by replacing the varnished interface with a pre-loaded spring that applies a constant force throughout the small range of thermal expansions and contractions associated with thermal cycling of the sample prism. In addition, a thin layer of thermally conductive grease is applied to the sensor to enhance the thermal conductivity of the sensor-sample prism interface. The current fixturing hardware leads to a much more repeatable, reliable, and long lasting interface between the sensor and sample prism, and ultimately to more accurate measurements of refractive index.

\subsection{Light source enhancements}

The third critical requirement for accurately measuring refractive index is knowledge of incident wavelength. The requirement on knowledge of wavelength is a function of the spectral dispersion $(\mathrm{dn} / \mathrm{d} \lambda)$ of the material being measured. For example, a typical value for the dispersion of $\mathrm{LiF}$ is $-0.00002 / \mathrm{nm}$ in the visible part of the spectrum. In other words, for $\mathrm{LiF}$, the accuracy of our knowledge of wavelength must be better than $0.5 \mathrm{~nm}$ so that the contribution to uncertainty in wavelength contributes less than 0.00001 to the overall uncertainty in refractive index. For more dispersive materials such as $\mathrm{ZnSe}$, the dispersion can be more like $0.0004 / \mathrm{nm}$ in regions where it is transparent but nearing an absorption feature. Therefore we need to have knowledge of wavelength at the $0.25 \mathrm{~nm}$ level in order to limit the contribution of wavelength uncertainty to our overall error budget to 0.0001 in absolute index.

\subsubsection{Grating monochromator calibration and navigation}

To select the incident wavelength, we use a vacuum grating monochromator bolted directly to the entrance port of our vacuum chamber. The monochromator can be tuned remotely via an RS-232 interface and the position read out (openloop) by a mechanical dial on the exterior of the monochromator housing which is incremented by the monochromator driver motor. The motor drives a lead screw and single point of contact tangent arm inside the monochromator through a vacuum rotary feedthrough. With the current grating ( 150 grooves $/ \mathrm{mm})$, the dial counter resolution is $0.8 \mathrm{~nm} /$ dial count.

Such an arrangement is susceptible to mechanical wear, however, and the open-loop control is not conducive to automated operation. During the course of the development of CHARMS, we performed a calibration of the dial counter readout using multiple orders of several lines from a tunable HeNe laser and found its output to be sufficiently accurate and repeatable for our purposes. Eventually however, we began to notice a degradation of our data quality and traced it back to a growing non-repeatability in the monochromator calibration. Eventually the dial counter had worn to the point of being useless; without it we had no means to determine a reference setting for the monochromator controller.

To solve this problem, we implemented an absolute optical encoder similar to those on our rotation stages on the grating shaft inside the monochromator (see Figure 3). This solution gave us not only a very accurate and repeatable knowledge of wavelength, but also absolute knowledge of wavelength without having to appeal to a reference position and the ability to tune wavelength in a closed-loop fashion completely under automated software control. 


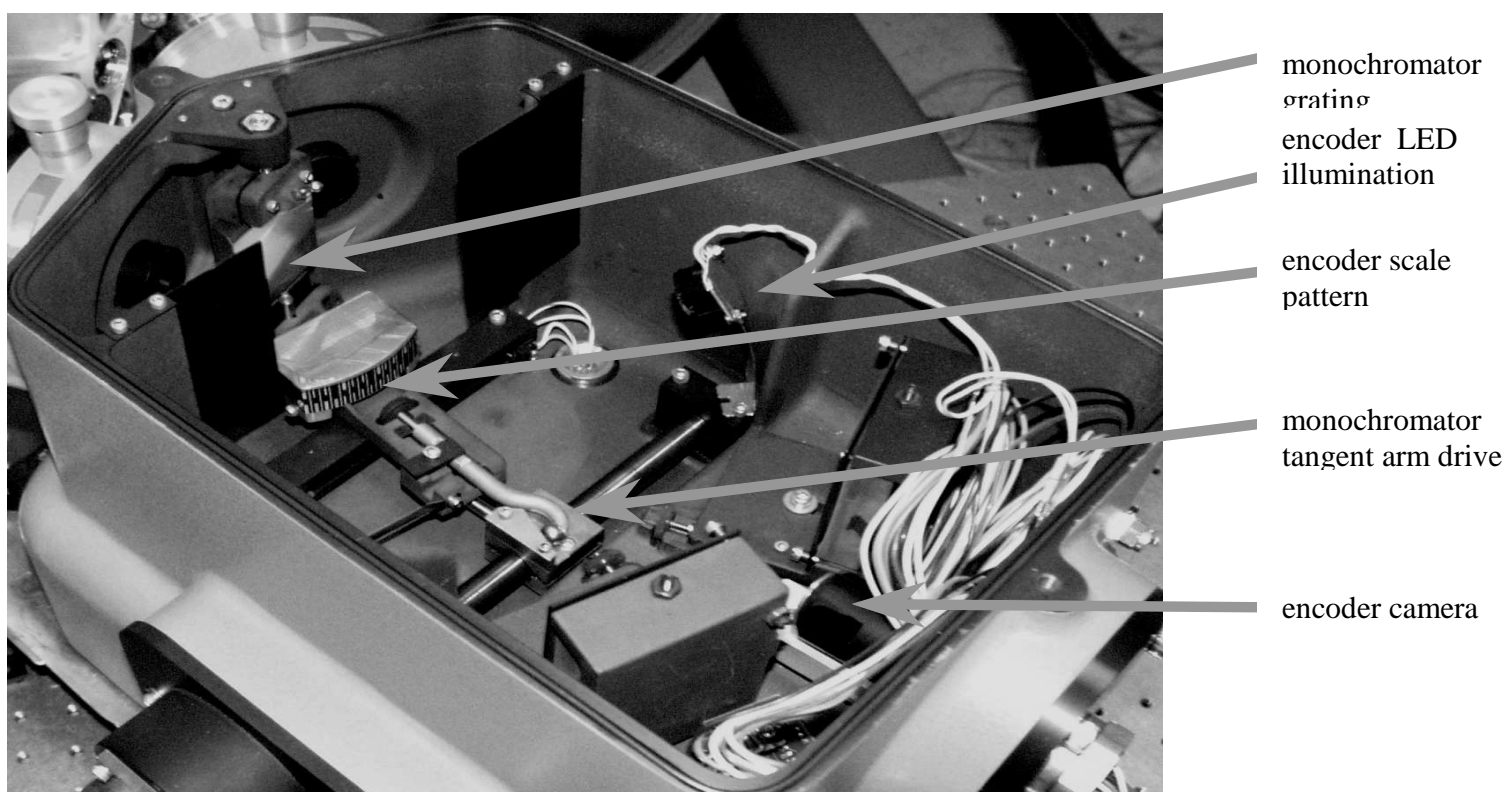

Figure 3: Absolute optical encoder adapted to report shaft angle of grating inside vacuum monochromator

Just as we had done for the monochromator dial counter, we calibrated the grating shaft encoder by recording the encoder reading associated with several different orders of multiple lines of our tunable HeNe laser. With the laser source installed in the monochromator, we would set the refractometer to observe the undeviated beam on our CCD detector. We then wrote a software routine that would slowly scan the monochromator through wavelength space and simultaneously record the reading on the grating encoder along with integrated flux from the slit image on the CCD. From this information, we were able to determine a centroid of the flux distribution and derive an encoder value associated with the precise $\mathrm{m} \bullet \lambda$ as defined by the HeNe laser. This process was repeated for $\mathrm{m} \bullet \lambda \mathrm{s}$ ranging from $1.7 \mu \mathrm{m}$ to $5.8 \mu \mathrm{m}$ which covers the entire range in $\mathrm{m} \bullet \lambda$-space required for us to perform our measurements since we use higher orders for short wavelengths to utilize the increased efficiency of the grating near the blaze wavelength $(4 \mu \mathrm{m})$. Based on these calibration values, we performed a $5^{\text {th }}$ order polynomial least squares fit that allows us to tune the monochromator grating accurately to within 20 arcsec of the desired calibration values (see Figure 4). This is good enough to give us sub-nm absolute wavelength accuracy in first order (and therefore a factor of 1/order \# better for higher orders) over our entire measurement range and has also allowed us to establish closed-loop wavelength control and even further automate the measurement process.

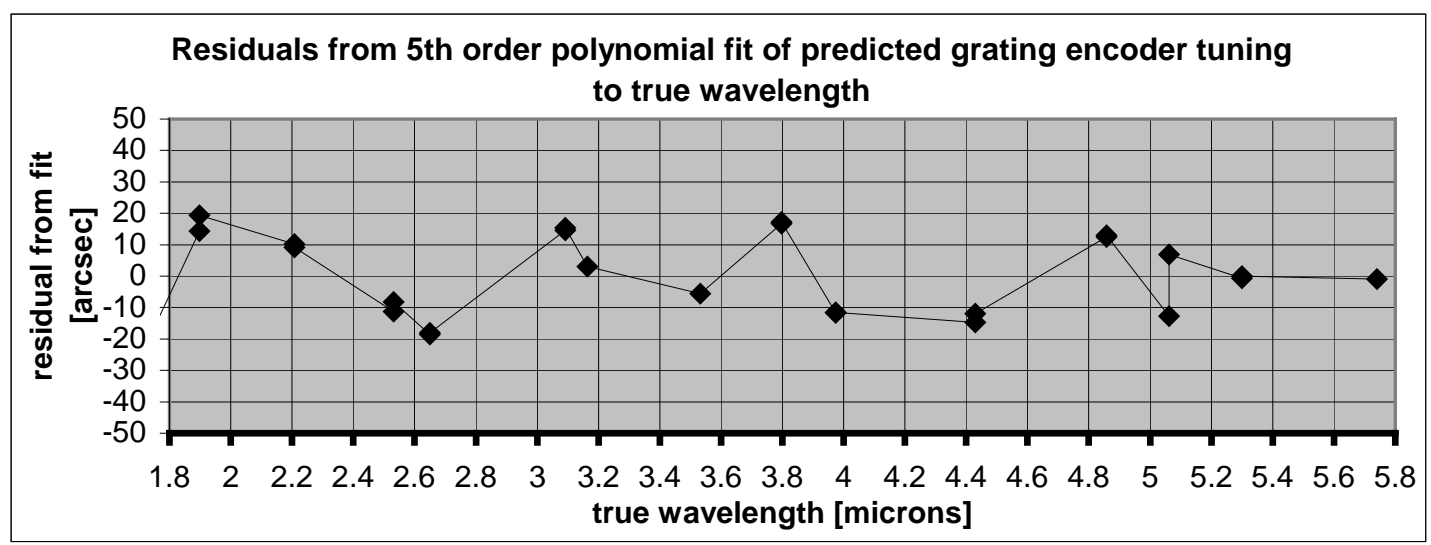

Figure 4: Residuals from fit of monochromator grating encoder wavelength calibration using tunable HeNe laser 


\subsubsection{Order sorting filter wheel}

The addition of an absolute encoder on the rotation shaft of the monochromator grating has allowed us to very accurately determine $\mathrm{m} \bullet \lambda$, but not necessarily $\lambda$ itself. Since we often work close to the grating blaze wavelength of $4 \mu \mathrm{m}$ to maximize grating efficiency, our data acquisition code would occasionally be confused by adjacent orders falling on an array detector at the same time and would pick the wrong one. A valid measurement would result - just not at the intended wavelength. In order to resolve this ambiguity we have designed and built a custom, 24-position order sorting filter wheel mechanism (see Figure 5). The filter plane lies just outside the entrance slit of the monochromator and is therefore not included in the evacuated volume of the monochromator. Populating the filter wheel are a variety of narrow band and long-pass filters depending on the order, wavelength, and detector(s) that will be used for measurements at that particular wavelength. To navigate between the 24 positions on the filter wheel we have implemented a modified Gray code encoder printed on paper and imaged by the same model CCD camera used in our other absolute encoders and illuminated by a bank of LEDs. The encoder scale is a modified Gray code pattern because it includes an additional track of fiducial lines outside the 5 position bits that act as detents to allow us to center the selected filter aperture over the monochromator's entrance slit.

The implementation of these upgrades to our monochromator source assembly has improved the quality of our data in several ways. First, we now have an accurate and repeatable knowledge of tuned wavelength to which to associate measured refractive index values. Second, by order sorting and removing the ambiguities that can arise from working near the blaze wavelength of the grating, we have far fewer rejected data points at undesired wavelengths. In addition, by working as near the blaze wavelength as possible, we have been able to increase our signal over background, reduce the exposure time required of our detectors, and therefore acquire data much more efficiently. Finally, all of the improvements have allowed us to fully automate the formerly manual wavelength selection and verification processes under software control.

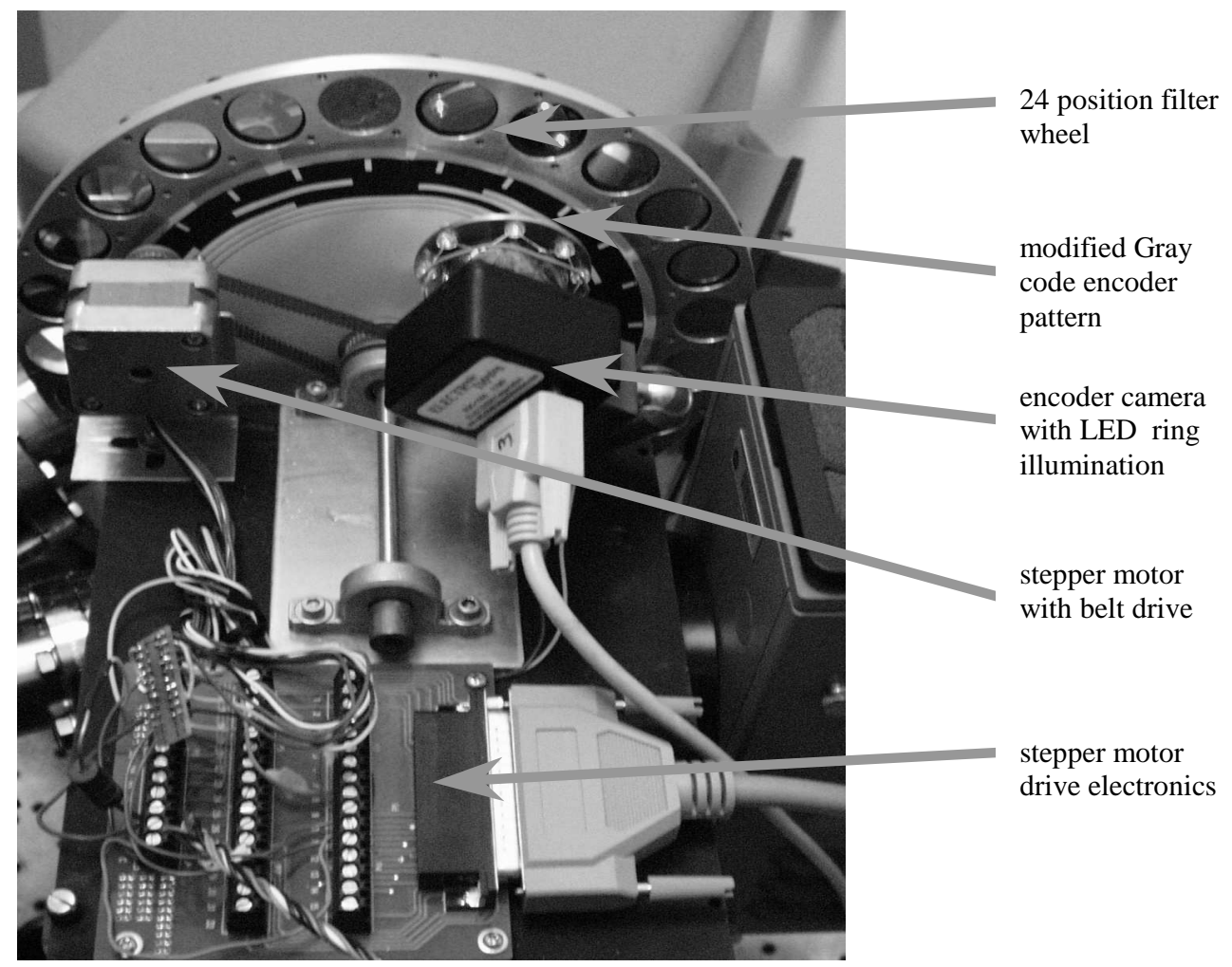

Figure 5: Custom 24-position filter wheel used for order sorting with modified Gray code encoder 


\section{MEASUREMENT PROCESS}

The measurement of a single sample specimen of a material over the full wavelength and temperature space ( $0.4 \mu \mathrm{m}$ to $5.6 \mu \mathrm{m}$ at $15 \mathrm{~K}$ to $300 \mathrm{~K}$ ) typically consumes 2 full weeks of measurements. This includes repetition of each type of measurement run at least twice on different days so that systematic faults will reveal themselves more readily. Such a dataset includes wavelength sampling at the $50 \mathrm{~nm}$ or 100-200 nm level in the visible or IR respectively and temperature sampling at about the $3 \mathrm{~K}$ level giving a total of 3000-4000 individual data points. Fortunately most of that data acquisition process has been automated and is performed entirely under software control. The flow chart in Figure 6 highlights steps that are required for each and every data point acquired by CHARMS.

\section{DATA REDUCTION}

Since we first began taking data with CHARMS, data acquisition code has written text data files suitable for spreadsheet based data reduction. Before we achieved high levels of automation, these files had byte counts of the order of $300 \mathrm{~KB}$. Now that the refractometer system is fully automated, files are typically about $3 \mathrm{MB}$ in size per 24 hours of continuous measurement. After analyzing data for even the first few data runs, it became clear that while it would take two weeks to collect the raw data, it would take three weeks to reduce the data by hand in a spreadsheet! This realization led to the development of a computer program written in Visual Basic we call the CHARMS Data Cruncher (CDC). CDC allows us to not only examine raw data from the refractometer to get in-process assessments of data quality, but ultimately it produces a list of fully validated, measured index values which can later be fit to Sellmeier equations. CDC follows the basic process outlined below to digest raw data acquisition products into measured refractive indices.

\subsection{Process File}

The user selects a raw data file for processing. CDC identifies the file format revision, which determines which detector was used for measurements and what auxiliary data was collected, and branches to appropriate routines to parse the selected data file in order to: a) detect the apex angle of the prism being measured which it uses in all of its computations of index based on the equation in Section 2.2 for index under condition of minimum deviation; b) derive the angle for the rotating fold, as described earlier, which corresponds to the slit image falling exactly at some reference position in the focal plane for each measurement of beam angle without regard to whether the beam is a deviated one or an undeviated one. Each reading is also tagged with a time and date stamp, an average sample temperature, the measurement wavelength and grating order number, the encoder reading for the monochromator's grating, and an $\mathrm{R}^{2}$ value indicating the quality of the fit for the least squares determination of the line through the ordered pairs of fold flat encoder readings and slit image centroid positions on the detector; and c) determine whether each reading represents a deviated beam or an undeviated beam and to segregate them into separate files accordingly. For deviated readings, CDC also produces a measure of the degree to which the minimum deviation condition has been maintained.

\subsection{Show Undeviated Readings}

The user can graphically display all undeviated readings as a group to view: a) an $\mathrm{R}^{2}$ value for each point which indicates whether all individual encoder readings and slit position centroid values are healthy. $\mathrm{R}^{2}$ values below 0.999 usually have some deficiency which can often be manually corrected so that each valuable data point can be healed. There are several common ailments from which data groups (which contain as many as 120 encoder readings, 120 temperature sensor readings, and 40 image centroid values) can suffer. Data group problems range in severity from negligible, to trivially corrected, to "nuisance to correct," to uncorrectable. An experienced user of CDC can often tell from the actual $\mathrm{R}^{2}$ value exactly what the problem is likely to be and how to deal with it; $b$ ) the general trending with time of the undeviated beam readings. One can usually tell when the temperature of the sample chamber is being rapidly cooled with cryogens as the measured angle of the undeviated beam will drift in a characteristic way as the changing thermal environment causes thermo-mechanical gradients which result in slight misalignments of the refractometer's optics. The undeviated beam can move as much as 10 arcseconds during a harsh thermal transient (Figure 7). The interpolation described in Section 4.4 below mitigates the effect of beam drift (which affects both undeviated and deviated beams) and makes the determination of deviations in this refractometer truly differential. Under steady state thermal conditions, undeviated beam readings can be stable at the $+/-0.2$ arcsecond level; and c) the plotted trajectory of the slit image across the image sensor or the integrated intensity of light passing through the detector slit as the fold mirror scans the image across the slit. Each point in a run can be examined individually to aid in determining the cause of a possible fault. 


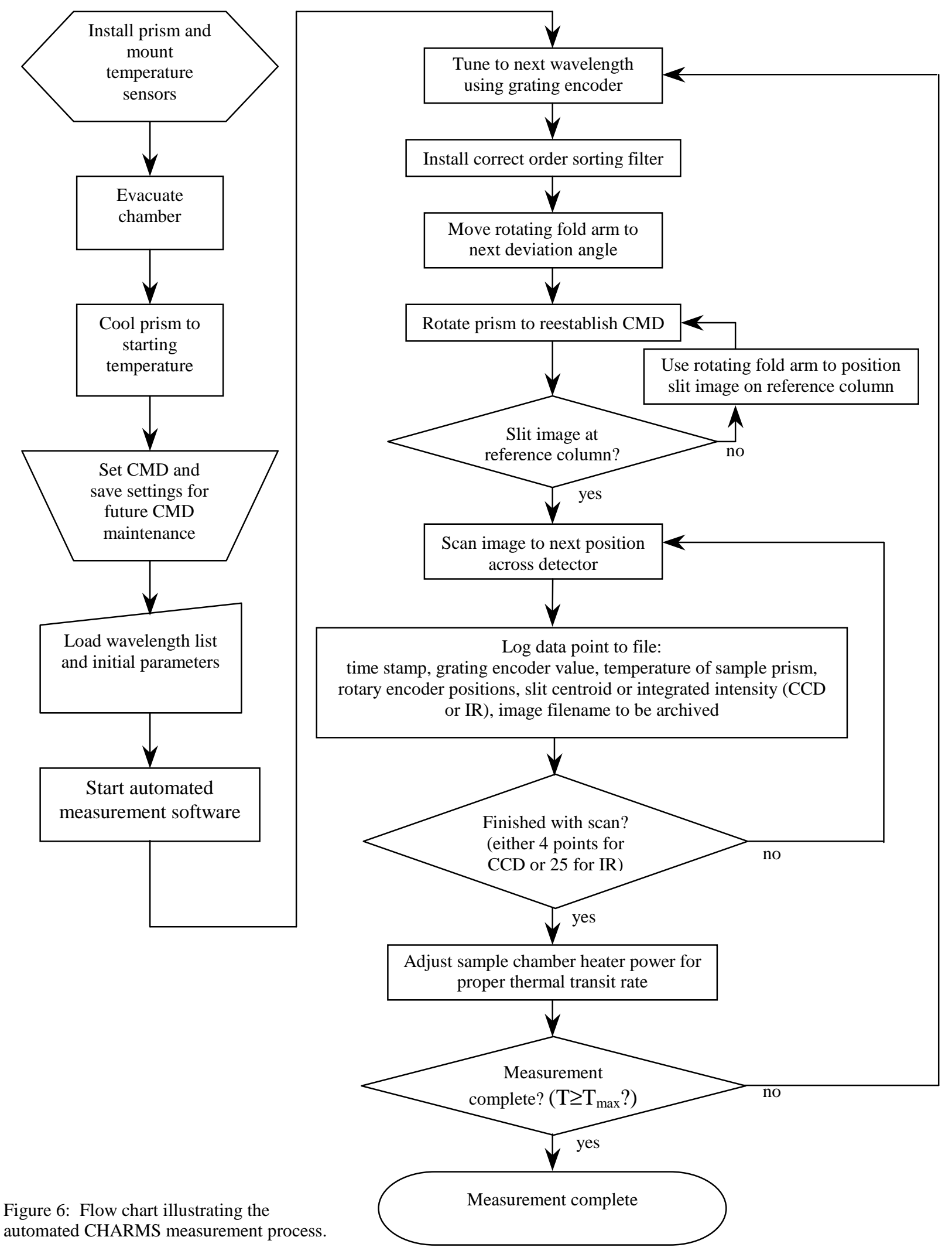




\subsection{Show Deviated Readings}

In addition to allowing the user to view the same things as for undeviated beams, the user can elect to graphically display all deviated readings as a group to see: a) the degree to which the condition of minimum deviation has been maintained; and $b$ ) the general trending of beam deviation due to dispersion in the material being measured as wavelength is repetitively tuned over a spectral region. An outlier point from one of the smooth sweeping curves for a given wavelength (Figure 8) is indicative of a problem with that point.

\subsection{Compute Index}

Once the user has approved the quality of all deviated and undeviated beam readings, CDC will automatically generate a file containing time/date/temperature-stamped, computed absolute refractive index values from the selected data run. In doing so, it computes beam deviations for each deviated reading taking the difference between the reading itself and an undeviated reading $\mathrm{U}\left(\mathrm{t}_{\mathrm{dev}}\right)$ which has been derived from undeviated readings $\mathrm{U}\left(\mathrm{t}_{\mathrm{dev}-1}\right)$ and $\mathrm{U}\left(\mathrm{t}_{\mathrm{dev}+1}\right)$ taken at times on either side of the deviated reading interpolated to the time of the deviated reading itself:

$$
\mathrm{U}\left(\mathrm{t}_{\mathrm{dev}}\right)=\mathrm{U}\left(\mathrm{t}_{\mathrm{dev}-1}\right)+\left(\mathrm{U}\left(\mathrm{t}_{\mathrm{dev}+1}\right)-\mathrm{U}\left(\mathrm{t}_{\mathrm{dev}-1}\right)\right) \cdot\left[\left(\mathrm{t}_{\mathrm{dev}}-\mathrm{t}_{\mathrm{dev}-1}\right) /\left(\mathrm{t}_{\mathrm{dev}+1^{-}} \mathrm{t}_{\mathrm{dev}-1}\right)\right]
$$

\subsection{Blend Index Files}

This routine simply produces a list which is the combined data from numerous data runs having a common root file name. The combined list is sorted by wavelength first and then by temperature within each wavelength grouping.

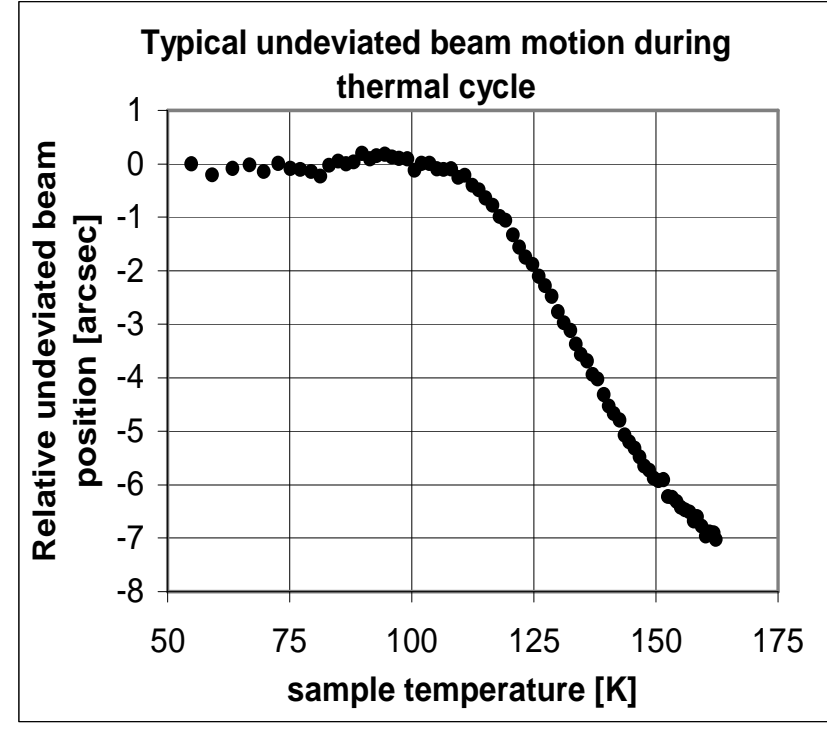

Figure 7- Typical relative position of the undeviated beam during a thermal cycle ( $~ 8$ hours). 1.5 arcsec relative motion, if unaccounted for, is enough to cause an error of $1 \cdot 10^{-5}$ in absolute index for the material being measured

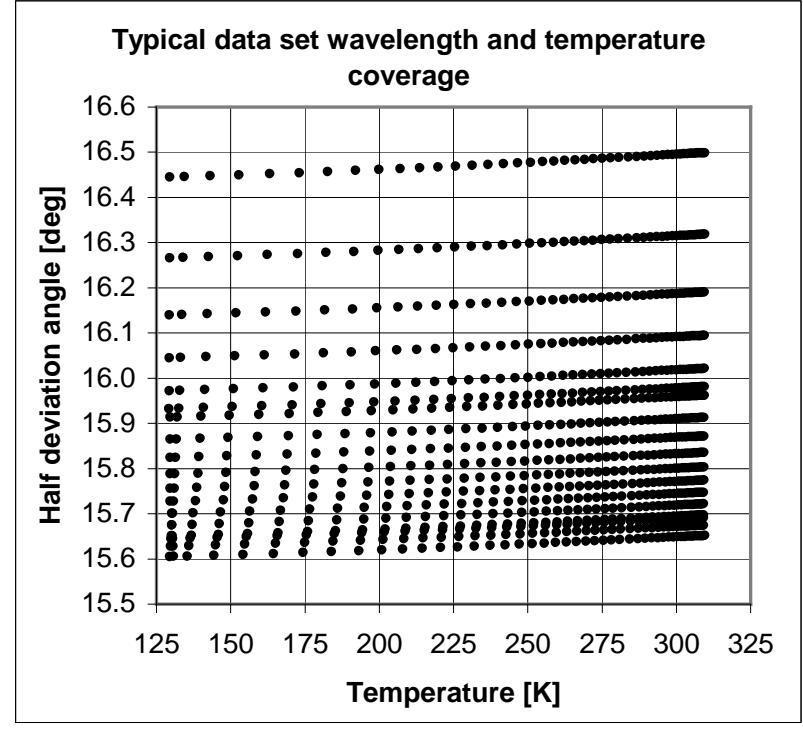

Figure 8: Typical wavelength and temperature coverage for a single data run. Each of the horizontal contours at nearly constant deviation angle corresponds to a different wavelength. The slope of these contours shows $\mathrm{dn} / \mathrm{dT}$ of the material over this temperature range.

\subsection{Fit Index(T)}

This routine fits measured index values for each wavelength to piecewise, second order polynomials in temperature, $\mathrm{T}$, above and below some selected crossover temperature. These high quality quadratic fits, made using the method of least squares, are of the form $n(T)=c_{2} T^{2}+c_{1} T+c_{0}$. The temperature range for each fit is further restricted to include only points above what we call the saturation temperature which is that temperature below which we can no longer definitively sense a change in index. We call the saturation value of index that index which we measure at and below the saturation temperature. All materials we have studied to date exhibit a saturation temperature at or below $65 \mathrm{~K}$. 
CDC computes spectral dispersion in the material by first tabulating index values on a regular wavelength/temperature grid from the piecewise quadratic fits described above. From that table, a new table of spectral dispersion, $\mathrm{dn} / \mathrm{d} \lambda$, is computed by dividing differences in index value, $\mathrm{n}$, by corresponding differences in wavelength, $\lambda$, for each temperature. Thermo-optic coefficient, $\mathrm{dn} / \mathrm{dT}$, is simply the first derivative of $\mathrm{n}(\mathrm{T})$ with respect to $\mathrm{T}$ or $\mathrm{dn}(\mathrm{T}) / \mathrm{dT}=2 \mathrm{c}_{2} \mathrm{~T}+\mathrm{c}_{1}$. CDC produces a table of thermo-optic coefficients on that same regular wavelength and temperature grid.

\subsection{Plot Index}

If all is well with the refractometer, this routine produces a plot like that in Figure 9 in which the agreement in index measurements at each wavelength over the same broad temperature range for certain samples is repeatable to within a few parts in the sixth decimal place of index over several subsequent days of measurements. However, even with all of the data validation and screening steps described above in place, still, sometimes problem data points slip through. Indeed, what usually escapes previous validation steps is groups of points which suffer from an epidemic due to some systematic pathogenic behavior of the refractometer which was not evident at the time measurements commenced. In fact, most of the problems solved by hardware improvements discussed previously were revealed by this routine in CDC. Now that those improvements have been implemented, it is rare to discover entire groups of points which are errant. Examples of things which have been readily revealed by this routine are: 1) CMD not being properly maintained; 2) the search of the detector image for the location of the slit image incorrectly locking onto the image from an order of another, unintended wavelength (before the order sorting filter wheel was installed); 3) aging of the thermal contact of the temperature sensors on the sample after a few days of thermally cycling the sample to cryogenic temperatures and back to ambient; the quality of the contact would age and provides errant readings of sample temperature (Figure 10).

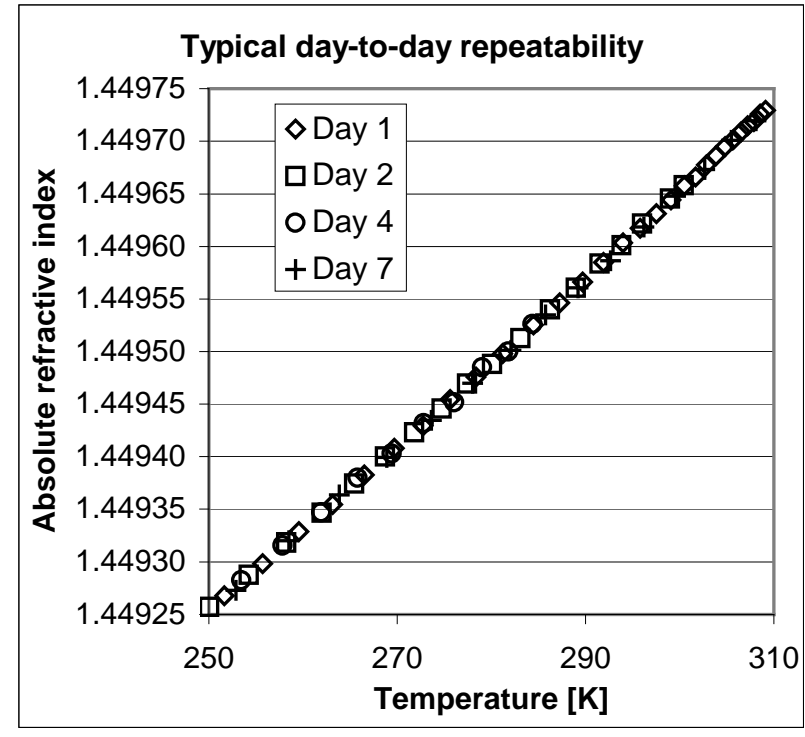

Figure 9 - typical repeatability near room temperature over multiple days of measurements. For this material our absolute accuracy is $1 \cdot 10^{-5}$ but our precision is nearly $2-3 x$ better.

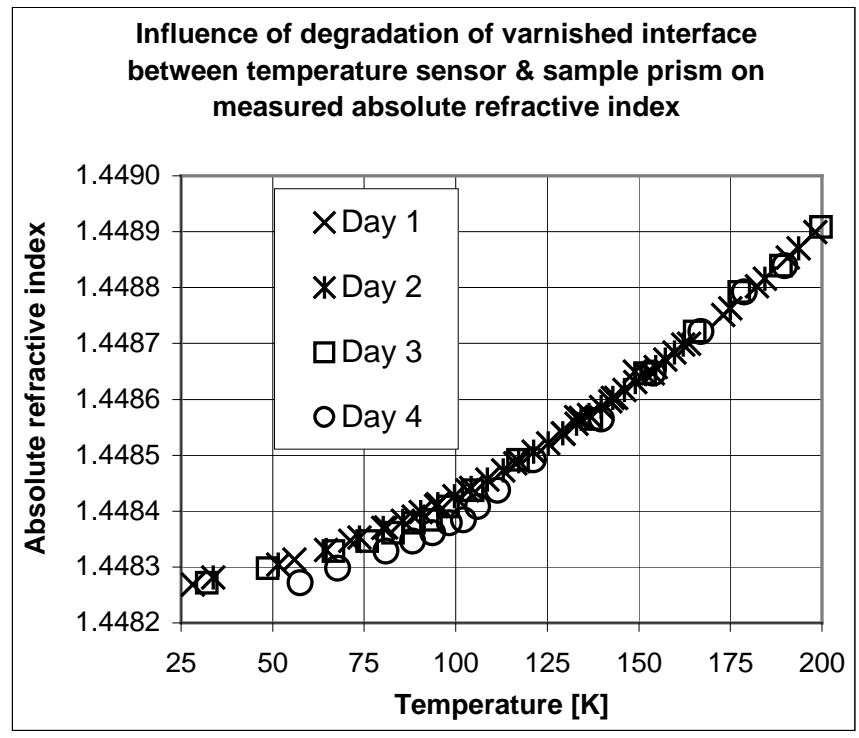

Figure 10: Typical degradation of measurement repeatability at cryogenic temperatures as a function of thermal cycling. The discrepancy at $52 \mathrm{~K}$ between Days 1 and 4 is $4 \cdot 10^{-5}$; absolute measurement accuracy for this material is $1 \cdot 10^{-5}$. The degradation of the sensor interface tends to manifest itself only at the lower temperatures (below $175 \mathrm{~K}$ in this example).

\subsection{Estimate Errors}

Finally, CDC produces a table of estimated index errors for different wavelength and temperature combinations. A partial index error $\mathrm{dn}$ is computed for each of four factors (based on presumably known uncertainties in those factors), and the four resulting dn's are combined in quadrature to produce a net index error estimate. The four partial dn's are computed using: 1) uncertainty in calibrated wavelength, $\mathrm{d} \lambda$, along with computed $\mathrm{dn} / \mathrm{d} \lambda ; 2$ ) uncertainty in measured temperature, dT, along with computed $\mathrm{dn} / \mathrm{dT} ; 3$ ) uncertainty in measured apex angle, $\mathrm{d} \alpha$, along with analytically derived $\mathrm{dn} / \mathrm{d} \alpha ;$ and 4 ) uncertainty in measured beam deviation angle, $\mathrm{d} \delta$, along with analytically derived $\mathrm{dn} / \mathrm{d} \delta$. 
Under conditions where flawless raw data files are available, the processing of a single day's data, which previously took as much as a day and half using spreadsheet methods, can be carried out with a few presses of a mouse button and a little examination of intermediate graphical data products in a couple of minutes time. When some aspect of our automated refractometer is misbehaving and generating errant data, it may take up to an hour to repair the raw data file, at the end of which time, the fault with the refractometer has almost always been identified so that it can be corrected before several more days are spent gathering compromised data.

\section{CONCLUSION}

Having overcome many engineering and technical challenges, CHARMS is finally a successfully operating, absolute, cryogenic refractometer. We have shown that our measured values of refractive index are in good agreement with literature values and our precision and repeatability are excellent. By virtue of carefully designed hardware upgrades we have been able to more fully automate the measurement process and to produce the highest quality data with very dense sampling in both wavelength and temperature on a reasonable time scale.

\section{REFERENCES}

${ }^{1}$ B.J. Frey, D.B. Leviton, Cryogenic high-accuracy absolute prism refractometer for infrared through far-ultra-violet optical materials: implementation and initial results," SPIE 5172, pp. 119-129, San Diego, August 2003

${ }^{2}$ D.B. Leviton, B.J. Frey, Thermal design considerations for the Cryogenic High Accuracy Refraction Measuring System (CHARMS)," SPIE 5172, pp. 25-35, San Diego, August 2003

${ }^{3}$ D.B. Leviton, B.J. Frey, "Cryogenic, High-Accuracy, Refraction Measuring System - a new facility for cryogenic infrared through far-ultraviolet refractive index measurements," SPIE $\mathbf{5 4 9 4}$ pp. 492-504, Glasgow, June 2004

${ }^{4}$ D.B. Leviton, B.J. Frey, "High-accuracy, absolute, cryogenic, refractive index measurements of infrared lens materials for JWST NIRCam using CHARMS," SPIE 5904-25, San Diego, 2005

${ }^{5}$ D.B. Leviton, B.J. Frey, "Ultra-high resolution, absolute position sensors for cryostatic applications," SPIE 4850, pp 776-787, Waikoloa, August 2002

${ }^{6}$ D.B. Leviton, B.J. Frey, "Design of a cryogenic, high accuracy, absolute prism refractometer for infrared through far ultraviolet optical materials," SPIE 4842, p. 259, Waikoloa, August 2002

${ }^{7}$ I. H. Malitson, "Interspecimen comparison of the refractive index of fused silica," J. of the OSA, 55(10), pp. 12051209, October 1965 